\title{
ESQUEMAS DE INCENTIVOS Y CARTERAS DE INVERSIÓN INNOVADORAS*
}

\section{INCENTIVE SCHEMES AND INNOVATIVE PORTFOLIOS}

\author{
GINO LOYOLA** \\ YOLANDA PORTILLA***
}

\begin{abstract}
Resumen
En el contexto de la administración delegada de carteras de inversión, este artículo caracteriza las propiedades que el esquema de compensación debe poseer para incentivar la selección de portafolios con alto riesgo y alto retorno esperado. En particular, se proporcionan condiciones que permiten esquemas remunerativos no monótonos que premian resultados extremos y castigan resultados moderados.

Palabras clave: Compensación ejecutiva; administración delegada de carteras; esquemas de incentivos no monótonos; propiedad de razón de verosimilitud no monótona.
\end{abstract}

\begin{abstract}
This article characterizes the properties of the compensation scheme of delegated portfolio management that would lead to the selection of high risk-high return portfolios. In particular, it provides conditions under which a non-monotone payment structure emerges as an optimal contract, which rewards extreme results and punishes moderate ones.
\end{abstract}

Key words: Executive compensation, delegated portfolio management, nonmonotone incentive schemes, non-monotone likelihood ratio property.

JEL Classification: D86, G11, G20, G30, J33, M52.

* Agradecemos los comentarios de Sigifredo Laengle y de dos árbitros anónimos, y la valiosa asistencia de investigación de Matías García. Cualquier error u omisión remanente es, por supuesto, de nuestra exclusiva responsabilidad.

** Departamento de Control de Gestión y Sistemas de Información, Universidad de Chile, Diagonal Paraguay 257, Of. 1303, Santiago, Chile. E-mail: gloyola@fen.uchile.cl, Tel.:+56(2) 97837 68, Fax: +56 (2 6351679$)$.

*** Facultad de Economía y Negocios, Universidad Diego Portales, Manuel Rodríguez Sur 253, Santiago, Chile. E-mail: yolanda.portilla@udp.cl, Tel.: (562) 67622 55, Fax: (562) 6762202 . 


\section{INTRODUCCIón}

Un importante hecho estilizado en la industria de la administración delegada de carteras son los magros retornos de la administración activa de estas inversiones, particularmente si se comparan con aquellos obtenidos por estrategias pasivas como, por ejemplo, un índice accionario (Malkiel, 1995; Gruber, 1996; Stracca 2006). En consecuencia, la administración activa de estas inversiones parece, paradójicamente, sustraer valor en lugar de agregarlo.

Esta inquietante evidencia ha despertado el interés por estudiar los esquemas de incentivos en el proceso de delegación de inversiones. En especial, durante las últimas dos décadas ha surgido una fructífera literatura de teoría de contratos que evalúa si las estructuras de compensación utilizadas en la práctica proveen los incentivos adecuados para la conformación de estrategias de inversión con mayores retornos esperados. Dado que este tipo de estrategias suelen ir acompañadas de una mayor variabilidad en los resultados, la asunción de riesgo por parte de administradores de fondos aversos al riesgo constituye, en este contexto, un elemento central en el diseño de un buen mecanismo de incentivos.

$\mathrm{Al}$ respecto, la literatura previa ha mostrado que esquemas de incentivos monótonos -aquellos que exhiben una relación estrictamente creciente entre desempeño de las carteras y compensación de los administradores- no favorecen la toma de riesgos (Stoughton, 1993; Admati y Pfleiderer, 1997). ${ }^{1}$ Este resultado ha llevado a examinar las propiedades de esquemas de incentivos no monótonos (y convexos) tales como el esquema de comisiones asimétricas -que premian buenas rentabilidades pero no castigan malos resultados- (Starks, 1987) y los contratos tipo opciones (Garcia, 2001; Goetzmann, Ingersoll y Ross, 2003; Carpenter, 2000). A priori, la convexidad que dichos esquemas introducen en el perfil de compensaciones ayudaría a contrarrestar la concavidad de la función de utilidad de administradores de fondos aversos al riesgo, favoreciendo de este modo la selección de carteras más riesgosas. No obstante, un análisis más profundo de los efectos de esta clase de esquemas remunerativos ha establecido dos resultados que debilitan este argumento de convexidad. En primer lugar, en el marco de los modelos teóricos elaborados para estudiar la administración de carteras, este tipo de esquemas de incentivos no corresponden a la estructura de compensación óptima. En segundo lugar, no es cierto que estos esquemas propicien siempre la toma de mayores riesgos. Es más, estas estructuras de compensación generan efectos adicionales que van en una dirección contraria a aquella propuesta por el argumento de convexidad, pudiendo llevar incluso a la asunción de menores riesgos (Carpenter, 2000; Chen y Pennacchi, 2009; Ross 2004).

Consecuentemente, el objetivo del presente artículo es caracterizar, en el contexto de la administración delegada de carteras de inversión, las propiedades

\footnotetext{
Esta conclusión se enmarca en un resultado más categórico aún establecido por aplicaciones de la teoría de contratos a la administración delegada de carteras: el denominado resultado de irrelevancia. Según este resultado, el aumento de la participación del agente en el retorno de la cartera administrada no provee los incentivos en términos de esfuerzo y asunción de riesgo que sí se verifican en la teoría de contratos estándar (Stoughton 1993, Admati y Pfleiderer 1997).
} 
que el esquema de compensación debe poseer para incentivar efectivamente la selección de estrategias de inversión innovadoras (carteras con mayor riesgo, pero con mayor rentabilidad esperada). Con este fin, construimos un modelo de relación de agencia cuyo resultado principal proporciona condiciones bajo las cuales puede ser óptimo introducir una no monotonicidad entre la remuneración de los ejecutivos y el desempeño de las carteras bajo su administración. Esta falta de monotonicidad puede ser tan severa que incluso puede llegar a ser óptimo premiar rentabilidades extremas, ya sean éstas suficientemente altas o bajas, y castigar (relativamente) rentabilidades moderadas. Luego, y contrariamente a los esquemas convexos de incentivos analizados por la literatura previa, el mecanismo óptimo que proponemos podría contemplar no sólo no castigar rentabilidades bajas, sino premiarlas. Aunque contraintuitivo, este "premio al fracaso" podría emerger como una característica del contrato óptimo en tanto este premio representa una promesa ex ante que alienta a los ejecutivos a asumir mayores riesgos.

Es importante destacar la contribución del presente trabajo a la caracterización de esquemas de incentivos óptimos en el proceso de la administración delegada de inversiones. En efecto, como concluye Stracca (2006), en general ésta es una literatura donde los resultados negativos tienden a prevalecer por sobre los constructivos. Este fenómeno se debe al hecho de que, en el caso de la administración de carteras, el agente debe decidir tanto respecto a su esfuerzo como al riesgo involucrado en los portafolios. Esta dificultad ha significado que la caracterización de un esquema de compensaciones óptimo para la administración delegada de inversiones en un ambiente con información asimétrica y bajo condiciones suficientemente generales, sea aún una tarea pendiente en la agenda de investigación de la teoría de contratos. Esto último es particularmente cierto en el contexto de un modelo clásico de riesgo moral. En efecto, los trabajos que han estudiado el esquema de incentivos óptimos en este contexto fundamentan sus conclusiones en modelos con simetría de información (Ou-Yang, 2003; Cadenillas, Cvitanic y Zapatero, 2007), modelos de decisión de un "experto" (Dybvig, Farnsworth y Carpenter, 2010; Li y Zhou, 2006) y/o modelos con funciones de utilidades muy específicas (Ou-Yang, 2003; Dybvig, Farnsworth y Carpenter, 2010). En contraste a toda esta literatura previa, nuestro trabajo logra modelizar esta dualidad esfuerzo-riesgo en el marco de un problema tradicional de riesgo moral bajo supuestos razonablemente generales, pudiendo arribar al diseño de un mecanismo óptimo que efectivamente aliente la conformación de estrategias de inversión más atractivas.

Además de la literatura de teoría de contratos aplicada a la administración delegada de carteras discutida previamente, este estudio se relaciona con al menos otras dos ramas de la economía de la información. En primer lugar, este artículo está emparentado con la literatura de teoría de contratos que ha caracterizado condiciones bajo las cuales es óptimo un esquema de incentivos monótono. En el caso en que el esfuerzo es una variable binaria, esta literatura (Holmström, 1979; Mirrlees, 1976) muestra que la monotonicidad de la estructura de compensación está garantizada por la propiedad de un likelihood ratio monótono (MLRP). Sin embargo, si el esfuerzo puede tomar más de dos valores, dicha monotonicidad requiere de una condición adicional, tal como la propiedad de una función de distribución cóncava en el esfuerzo (CDFP) (Mirrlees, 1979), o 
de una condición más fuerte, tal como la denominada spanning condition (SC) (Grossman y Hart, 1983). ${ }^{2}$

En segundo lugar, nuestro trabajo tiene también conexiones lógicas con la literatura que admite no monotonicidades en los esquemas de incentivos en contextos distintos al de la administración de carteras de inversión. La primera referencia nos remite a Grossman y Hart (1983), quienes proporcionan un ejemplo en que la monotonicidad de la estructura óptima de incentivos no se verifica debido al incumplimiento de SC (incluso aunque la condición de MLRP se satisface). Para estos autores, esta no monotonicidad surge por el doble papel que juega el producto (u otra medida de desempeño del agente) sobre el beneficio del principal: uno que permite aumentar su consumo y otro que permite mejorar su información respecto del esfuerzo no observado. En algunas circunstancias, ambos roles pueden entrar en conflicto, lo que podría llevar a la optimalidad de premiar resultados malos respecto de resultados moderados. Por su parte, Innes (1990) también admite la posibilidad de no monotonicidades en el contrato de financiamiento óptimo entre un inversionista y un emprendedor (un contrato del tipo "live-or-die"). Sin embargo, en este caso la fuente de dicha no monotonicidad es la presencia de cláusulas de responsabilidad limitada para ambas partes del contrato, y no el incumplimiento de la MLRP. Similarmente, el trabajo de Heinkel y Stoughton (1994) deriva un esquema de premios no monótono en el que resultados demasiado buenos implican el reemplazo del agente. No obstante, mientras sus hallazgos se refieren a la solución de un problema de selección adversa, el esquema propuesto en el presente artículo resuelve un problema de riesgo moral. Por último, Hvide (2002) caracteriza un contrato óptimo que incluye no monotonicidades, pero en el que no se requiere la violación de la MLRP. Sin embargo, mientras sus conclusiones son alcanzadas en el contexto de un modelo de teoría de torneos (evaluación de desempeño relativo), nuestros resultados son derivados en un modelo clásico de riesgo moral.

Es importante señalar que, no obstante, en general todos estos estudios consideran a la no monotonicidad una propiedad no deseable de los esquemas de compensación (porque no parece realista) y, en consecuencia, introducen restricciones de monotonicidad al programa que resuelve el contrato óptimo. Contrariamente a esta literatura, nuestro trabajo argumenta que en la industria de la administración delegada de inversiones la no monotonicidad puede ser una propiedad deseable (para incentivar la toma de riesgos y la innovación en la selección de portafolios), e incluso realista (por el antecedente de prácticas compensatorias no monótonas observadas en los mercados financieros). De este modo, no sólo permitimos la posibilidad de esquemas de compensación no monótonos, sino además caracterizamos las condiciones bajo las cuales un esquema de este tipo emerge como óptimo.

La estructura del artículo es la siguiente. La sección 2 presenta un ejemplo que ilustra una situación en que el esquema óptimo de incentivos admite severas

2 En el caso en que el esfuerzo es una variable continua, SC también constituye una condición suficiente para un esquema óptimo de incentivos monótono (ver Caillaud y Hermalin, 2000, sección 5). En el caso en que el resultado es una variable continua, las condiciones que garantizan esta monotonicidad no se ven alteradas (ver Caillaud y Hermalin, 2000, sección 4.5). 
no monotonicidades. La sección 3 desarrolla un modelo base de administración delegada de carteras de inversión bajo riesgo moral, en el que el trader puede escoger entre dos niveles de esfuerzo. La sección 4 caracteriza formalmente las condiciones bajo las cuales el contrato óptimo puede inducir un esquema de compensación que premie resultados extremos en este modelo base. La sección 5 explora el caso con un espacio continuo de niveles de esfuerzo, mostrando la robustez de los resultados hallados en el modelo con esfuerzo binario. Finalmente, la sección 6 discute las principales conclusiones. Las pruebas de los resultados principales están contenidas en el Anexo.

\section{UN EJEMPLO}

Esta sección ilustra las condiciones bajo las cuales el esquema de compensación óptimo puede incluir una relación severamente no monótona respecto al desempeño. Con este objeto, considere el siguiente ejemplo. Existe un proceso de delegación de administración de carteras de inversión bajo información asimétrica entre el dueño del fondo de inversión (principal) y el trader (agente). Las preferencias del trader dependen de $e$, el esfuerzo ejercido por conformar una cartera de inversión determinada, y de $w$, la compensación recibida por esta tarea. En particular, estas preferencias están representadas por la función de utilidad (ex post) dada por $U(w, e)=w^{1 / 2}-e^{2}$. El trader puede escoger entre dos niveles de esfuerzo: $e \in\{1,3\}$. Estos niveles de esfuerzo están relacionados unívocamente con dos carteras de inversión distintas: mientras $e=3$ permite conformar la cartera $A, e=1$ permite conformar la cartera $B$. El retorno de la cartera $k$ cuando ocurre el estado de la naturaleza $i$, denotado por $x_{i}^{k}$, puede tomar tres valores posibles tal que $x_{i}^{k} \in\{-1000,0,1500\}$ para todo $k=A, B$. La probabilidad de que la cartera $k$ genere un retorno $x_{i}$ es denotada por $p_{i}^{k}$. Estas probabilidades condicionadas están descritas por la siguiente tabla:

\begin{tabular}{|lccc|}
\hline$p_{i}^{k}$ & $x_{1}=-1000$ & $x_{2}=0$ & $x_{3}=1500$ \\
\hline$k=A$ & $2 / 5$ & $1 / 5$ & $2 / 5$ \\
$k=B$ & $1 / 6$ & $4 / 6$ & $1 / 6$ \\
\hline
\end{tabular}

El nivel de utilidad de la mejor de las alternativas descartadas por el trader al aceptar el contrato (utilidad de reserva) corresponde a $\underline{U}=0$. Por su parte, las preferencias del principal están representadas por la función de utilidad (ex post $)$ dada por $B\left(x_{i}, w\right)=x_{i}-w$, para todo $i$.

Con el objeto de caracterizar el esquema óptimo de incentivos, por ahora suponemos -lo que demostraremos más adelante- que el dueño del fondo de inversión desea que el trader seleccione la cartera $A$. En un contexto de asimetría de información, la decisión del trader respecto del nivel de esfuerzo -y por tanto 
respecto de la cartera de inversión- no es verificable por parte del principal, pero sí lo es el retorno de la inversión. Luego, si definimos $w_{i} \equiv w\left(x_{i}\right)$, la compensación pagada por el dueño del fondo mutuo al trader cuando se observa el retorno $x_{i}$, el programa que determina el contrato óptimo bajo información asimétrica corresponde a:

$$
\underset{\left\{w_{i}\right\}_{i=1}^{3}}{\operatorname{Max}} \sum_{i=1}^{3} p_{i}^{A}\left(x_{i}-w_{i}\right)
$$

s.a.

$$
\begin{gathered}
\sum_{i=1}^{3} p_{i}^{A} w_{i}^{1 / 2} \geq 9 \\
\sum_{i=1}^{3} p_{i}^{A} w_{i}^{1 / 2}-9 \geq \sum_{i=1}^{3} p_{i}^{B} w_{i}^{1 / 2}-1 .
\end{gathered}
$$

La inecuación (2.1) representa la restricción de participación y la inecuación (2.2) corresponde a la restricción de compatibilidad de inventivos. Reemplazando las probabilidades, note que este problema es equivalente al siguiente programa:

$$
\begin{gathered}
\underset{\left\{w_{i}\right\}_{i=1}^{3}}{\operatorname{Min}}\left[\frac{2}{5} w_{1}+\frac{1}{5} w_{2}+\frac{2}{5} w_{3}\right] \\
\text { s.a } \\
\frac{2}{5} w_{1}^{1 / 2}+\frac{1}{5} w_{2}^{1 / 2}+\frac{2}{5} w_{3}^{1 / 2} \geq 9
\end{gathered}
$$

Del sistema de ecuaciones conformado por la restricción de participación (2.3) y la restricción de compatibilidad de incentivos (2.4) activas, es posible calcular que: ${ }^{3}$

$$
\begin{gathered}
w_{3}=345,4641-37,172 w_{1}^{1 / 2}+w_{1}, \\
w_{2}{ }^{*}=61,6539 .
\end{gathered}
$$

3 El hecho de que ambas restricciones estén activas en el óptimo es un resultado clásico de la teoría de contratos (Ver, por ejemplo, Bolton y Dewatripont, 2005). 
Sustituyendo en la función objetivo, tenemos que el programa original puede ser reducido a:

$$
\underset{w_{1}}{\operatorname{Min}}\left(2 w_{1}-37,172 w_{1}^{1 / 2}\right)
$$

De la condición de primer orden de este problema, obtenemos que $w_{1}^{*}=86,3598$ Tras reemplazar en (2.5) encontramos finalmente que $w_{3}^{*}=86,3598$.

Ahora comprobamos si efectivamente el dueño del fondo prefiere la cartera $A$ en lugar de la $B$. Para ello, verificamos que: ${ }^{4}$

$$
\sum_{i=1}^{n}\left(p_{i}^{A}-p_{i}^{B}\right) x_{i}=116,7>80,42=\sum_{i=1}^{n} p_{i}^{A} w_{i}^{*}-w^{*}(e=1),
$$

donde $w^{*}(e=1)=1$ corresponde al salario óptimo bajo simetría de información cuando el principal desea que se seleccione la cartera $B$, o equivalentemente, que el trader ejerza un nivel de esfuerzo $e=1.5$

Es claro entonces que el principal prefiere que el agente seleccione la cartera $A \mathrm{y}$, en consecuencia, el contrato óptimo está caracterizado por el siguiente esquema remunerativo:

$$
w^{*}(x)=\left\{\begin{array}{lll}
86,3598 & \text { si } & x=-1000 \\
61,6539 & \text { si } & x=0 \\
86,3598 & \text { si } & x=1500
\end{array}\right. \text {. }
$$

Luego, la relación entre el esquema compensatorio del trader y los retornos observados es claramente no monótona. Como lo comprobaremos del análisis formal desarrollado en la sección 4, esta característica particular del contrato óptimo es el resultado de la violación de una propiedad estadística recurrente en los modelos teóricos de riesgo moral. ${ }^{6}$ Por ahora, podemos decir que en este ejemplo no está garantizado que sea siempre menos probable observar malos resultados cuando se selecciona la cartera $A$ en vez de la $B$ (por ejemplo, $p_{1}^{A}=2 / 5>1 / 6=p_{1}^{B}$ ). Esto lleva entonces a "premiar" también una rentabilidad baja como $x_{1}=-1000$, al ser más probable que ésta provenga de la cartera $A$.

\section{El Modelo Base}

En esta sección construimos un modelo que formaliza y generaliza los resultados ilustrados por el ejemplo descrito en la sección anterior. Con este objeto, considere la siguiente relación de agencia entre el dueño de un fondo de inversión

\footnotetext{
Esta condición es formalizada en la Proposición 2, sección 4.

Este salario es computado en el Anexo B.

6 Esta propiedad corresponde a la denominada monotone likelihood ratio property (MLRP).
} 
(principal) y el administrador de dicho fondo (agente). ${ }^{7}$ El principal es neutral al riesgo tal que sus preferencias están representadas por la función de utilidad (ex post) $B\left(x_{i}, w\right)=x_{i}-w \cdot{ }^{8}$ Por su parte, las preferencias del agente están descritas por la siguiente función de utilidad (ex post) aditivamente separable:

$$
U(w, e)=u(w)-v(e)
$$

donde $e$ el nivel de esfuerzo asociado a la conformación de carteras de inversión y $w$ la compensación recibida por dicha tarea. La función $u(\cdot)$ representa el efecto sobre el bienestar del agente debido a su compensación, y satisface $u_{w}^{\prime}>0, u_{w}^{\prime \prime}<0$. La primera condición significa que el bienestar del agente siempre aumenta con la compensación (utilidad marginal positiva), y la segunda condición garantiza que el agente es averso al riesgo (utilidad marginal decreciente). Por otra parte, la función $v(\cdot)$ representa la desutilidad que el ejercicio de esfuerzo supone para el agente. Al respecto, suponemos que esta función satisface $v_{e}^{\prime}>0$ (desutilidad marginal positiva) $\mathrm{y} v_{e}^{\prime \prime}>0$ (desutilidad marginal creciente). La utilidad de reserva del agente corresponde a $\underline{U}$.

El trader puede escoger entre dos carteras de activos financieros distintas, $A$ y $B$. Dado que asumimos que la cartera $A$ presenta un mayor retorno esperado y una mayor volatilidad que la cartera $B$, decimos que el primer portafolio es más "innovador" que el segundo. Debido a que suponemos que la elección de la cartera $B$ puede ser entendida como simplemente replicar la cartera de mercado, asumimos que el nivel de esfuerzo asociado a ella es inferior a aquél necesario para conformar la cartera $A$. Este supuesto lo materializamos asumiendo que el agente puede escoger entre dos niveles de esfuerzo, cada uno de ellos relacionado unívocamente con uno de los dos portafolios de inversión. Por tanto, suponemos que $e \in\left\{e_{h}, e_{l}\right\}$, con $e_{h}>e_{l}, e_{j} \geq 0$ para todo $j=h, l$ y $v\left(e_{h}\right)>v\left(e_{l}\right)$. De este modo, mientras $e_{h}$ es el nivel de esfuerzo que permite conformar la cartera $A, e_{l}$ permite seleccionar la cartera $B$. Por otra parte, asumimos que $I$ corresponde al monto de recursos delegados al trader de acuerdo a un criterio predeterminado $\mathrm{y}$, por tanto, constituye una variable exógena del modelo.

7 Suponemos que el administrador del fondo es el mismo que el trader. Esto es equivalente a asumir que no existen problemas de agencia entre ambos.

8 El supuesto de neutralidad al riesgo por parte del principal se basa en la idea de que el dueño del fondo de inversión tiene la posibilidad de diversificar el riesgo mediante la distribución de su riqueza en distintos fondos e instrumentos financieros adecuadamente correlacionados. Por tanto, aunque originalmente el dueño del fondo puede ser averso al riesgo, su actitud frente al riesgo se asemeja a la de un individuo neutral al riesgo tal que sus preferencias dependen fundamentalmente del retorno esperado de las carteras. Al respecto, como indica Stracca (2006), en general la literatura teórica de administración delegada de carteras ha adoptado este mismo supuesto. Note que en un contexto en que la aversión al riesgo es decreciente con la riqueza, asumir neutralidad al riesgo por parte del principal puede ser especialmente plausible para inversionistas que poseen un nivel de riqueza suficientemente alto. Para una discusión referente a los efectos que este supuesto puede tener sobre los resultados del presente artículo, ver subsección 4.2. 
Las decisiones de esfuerzo y de selección de carteras no son observadas (o no son verificables) por el dueño del fondo. Sin embargo, éste es capaz de verificar las rentabilidades de ambas carteras. Sea $x_{i}^{k}$ el retorno generado por la cartera $k$ cuando ocurre el estado de la naturaleza $i$. Suponemos que $x_{i}^{k}$ puede tomar $n$ valores posibles tal que $x_{i}^{k} \in X=\left\{x_{1}, \ldots, x_{n}\right\}$ para todo $k=A, B$. Por simplicidad, asumimos que $x_{1}<x_{2} \ldots<x_{n}$. Denotamos por $p_{i}^{k}$ a la probabilidad de ocurrencia del retorno $x_{i}^{k}$, tal que se verifica que $p_{i}^{k}>0$ para todo $i, k$, y $\sum_{i=1}^{n} p_{i}^{k}=1$. $^{9}$

Además, asumimos que el retorno esperado de ambas carteras es suficientemente alto tal que $\sum_{i=1}^{n} p_{i}^{k} x_{i}^{k}-I>0$ para todo $k$. Este supuesto garantiza que el valor presente neto esperado (asumiendo una tasa de descuento igual a cero) asociado a ambas carteras es positivo, y por tanto, queda descartada la posibilidad de invertir en carteras que $a$ priori pueden ser calificadas como "fracasadas". ${ }^{10}$ $\mathrm{Si}$, por el contrario, permitiésemos esta clase de carteras, un retorno demasiado bajo podría ser altamente informativo de un esfuerzo muy bajo y, por ende, el esquema de incentivo óptimo no necesariamente premiaría desempeños deficientes. Por consiguiente, este supuesto es consistente con situaciones en que es óptimo premiar el fracaso ex post, pero descarta la posibilidad de premiar el fracaso ex ante. ${ }^{11}$

Es importante notar que entendemos el marco analítico desarrollado en este artículo como un modelo de referencia a partir del cual se puedan agregar los efectos de otros elementos que influyen sobre los contratos de ejecutivos, tales como la selección adversa, los contratos incompletos o el career concern. Desde nuestro punto de vista, el modelo más simple (e intuitivo) que permite contar con dicho benchmark es un modelo clásico de riesgo moral. Por cierto, este modelo es sólo un primer paso y está lejos de pretender ser una respuesta definitiva al diseño de esquemas compensatorios óptimos que incentiven la selección de carteras de inversión innovadoras. En consecuencia, reconocemos las limitantes de nuestro análisis y la necesidad de someter la robustez de nuestros resultados a extensiones que consideren elementos más realistas en el modelamiento de los esquemas de incentivos de ejecutivos.

9 Note que $p_{i}^{k}$ corresponde a la probabilidad de que el retorno observado sea $x_{i}$ condicional a que la cartera escogida por el trader (decisión no observada) es $k$.

10 Agradecemos a un árbitro anónimo por ayudarnos a clarificar los alcances de este supuesto.

11 No obstante, este supuesto no descarta la posibilidad de seleccionar carteras con niveles "excesivos" de riesgo. Como se discute en la sección Conclusiones, este fenómeno aparece como una extensión natural del modelo para ser abordada en futuros trabajos. 


\section{Resultados}

\subsection{Condiciones para un esquema de incentivos no monótono}

En esta sección caracterizamos el esquema de incentivos óptimo bajo el ambiente de asimetría de información descrito anteriormente. Para ello, procedemos en dos etapas. En primer lugar, suponemos que el dueño del fondo de inversión desea que el trader seleccione la cartera $A$. Bajo este supuesto, encontramos las condiciones que posibilitan una relación no monótona en el esquema óptimo de compensación (Proposición 1). En segundo lugar, caracterizamos las condiciones que garantizan que el dueño del fondo de inversión prefiere efectivamente la cartera $A$ en lugar de la $B$ (Proposición 2).

De este modo, bajo el supuesto de que el dueño del fondo de inversión prefiere la cartera $A$, el programa que determina el contrato óptimo está dado por: ${ }^{12}$

$$
\begin{gathered}
\underset{\left\{w\left(x_{i}\right)\right\}_{i=1}^{n}}{\operatorname{Max}} \sum_{i=1}^{n} p_{i}^{A}\left(x_{i}-w\left(x_{i}\right)\right) \\
\text { s.a. } \\
\sum_{i=1}^{n} p_{i}^{A} u\left(w\left(x_{i}\right)\right)-v\left(e_{h}\right) \geq \underline{U} \\
\sum_{i=1}^{n}\left(p_{i}^{A}-p_{i}^{B}\right) u\left(w\left(x_{i}\right)\right) \geq v\left(e_{h}\right)-v\left(e_{l}\right)
\end{gathered}
$$

donde (4.2) y (4.3) corresponden a la restricción de participación y restricción de compatibilidad de incentivos, respectivamente. Previo a enunciar nuestro principal resultado, necesitamos la siguiente definición.

Definición 1. Definimos $L R_{i}$, el coeficiente de verosimilitud (likelihood ratio), como

$$
L R_{i}=\frac{p_{i}^{A}}{p_{i}^{B}}
$$

para todo $i=1, \ldots, n$.

Según esta definición, $L R_{i}$ indica qué tan buena señal es el resultado $x_{i}$ del hecho de que el trader haya seleccionado la cartera $A$ (equivalentemente, que haya ejercido un nivel de esfuerzo $e_{h}$ ). Es decir, $L R_{i}$ refleja qué tan informativo es el retorno del fondo $x_{i}$ (observado) respecto de la decisión de selección de una cartera de inversión (no observada o no verificable). Luego, cuanto mayor sea $L R_{i}$, es más probable que el agente haya realizado un esfuerzo $e_{h}$ consistente

12 Note que excluimos de la función objetivo del principal la inversión I por ser ésta una variable exógena. 
con la cartera $A$. Por tanto, si el principal desea que esa cartera sea elegida, la compensación debiese ser más alta si se observa el retorno $x_{i}$.

Sin embargo, note que esto no garantiza que el esquema compensatorio será creciente en el resultado. Para ello se requiere que el coeficiente de verosimilitud sea monotónicamente creciente en el resultado $x_{i}$, propiedad conocida en la literatura de teoría de contratos como "monotone likelihood ratio property" (MLRP). El posible incumplimiento de esta propiedad nos permite precisamente arribar al siguiente resultado.

Proposición 1. Si existe un par de retornos $x_{i}$ y $x_{i+1}$ tal que $L R_{i} \geq L R_{i+1}$, entonces $w\left(x_{i}\right) \geq w\left(x_{i+1}\right)$.

Esta proposición nos provee una condición suficiente para la presencia de alguna no monotonicidad en el esquema de compensación óptimo del trader. Por lo tanto, si la MLRP creciente no se satisface, no podemos descartar la optimalidad de premiar no sólo altas, sino también bajas rentabilidades.

Este último fenómeno podría ser especialmente factible en la industria financiera si, como lo hemos supuesto, la cartera $A$ constituye una estrategia de inversión más innovadora que la cartera $B$. En efecto, es usual que esta mayor innovación traiga consigo mayores retornos esperados, pero también mayor volatilidad. ${ }^{13}$ Por tanto, es posible que resulte óptimo para el dueño del fondo de inversión premiar al trader cuando se observen resultados extremos (ya sean suficientemente altos o suficientemente bajos) y castigarlo (en términos relativos) cuando se observen resultados intermedios. De este modo, puede resultar eficiente "premiar el fracaso", en la medida que retornos deficientes sean suficientemente indicativos de un mayor esfuerzo y creatividad por formular estrategias de inversión con un mejor perfil de riesgo-retorno esperado. ${ }^{14}$

Por cierto, para que un esquema de incentivos con esta característica tan particular sea óptimo desde el punto de vista del dueño del fondo se requieren ciertas condiciones. Estas últimas se refieren a las propiedades de las distribuciones de probabilidad de los retornos de ambas carteras y a los costos salariales asociados a la selección de éstas. Estas condiciones son sintetizadas y formalizadas en el siguiente resultado.

Proposición 2. El dueño del fondo preferirá la cartera A sobre la cartera B si se verifica la siguiente condición:

$$
\sum_{i=1}^{n}\left(p_{i}^{A}-p_{i}^{B}\right) x_{i}>\sum_{i=1}^{n} p_{i}^{A} w^{*}\left(x_{i}\right)-w^{*}\left(e_{l}\right) .
$$

13 Utilizando la terminología financiera, podemos decir que la cartera $A$ es una inversión más activa que la cartera $B$ en el sentido de que la primera se aleja más de un portafolio de referencia que la segunda.

14 La pertinencia de un "premio al fracaso" ha sido también planteada para estimular procesos de innovación y exploración tecnológica. Véase, por ejemplo, Manso (2010). 
Este resultado sugiere que una condición necesaria para que el dueño del fondo prefiera la cartera $A$ es que su retorno esperado supere a aquél de la cartera $B .{ }^{15}$ Sin embargo, se requiere además que esta diferencia en retornos esperados supere el mayor costo salarial que significa para el dueño del fondo inducir la selección de la cartera $A$ en lugar de la $B$. A su vez, este mayor costo salarial depende positivamente de tres factores: (i) la mayor desutilidad que significa ejercer un esfuerzo alto dada por la diferencia $v\left(e_{h}\right)-v\left(e_{l}\right)$, (ii) la volatilidad de la cartera $A$, y (iii) el grado de aversión al riesgo del trader.

Por lo tanto, no basta con que la cartera $A$ sea más innovadora que la cartera $B$, de tal modo que en promedio genere un mayor retorno. Se necesita además que ese mayor retorno esperado exceda los mayores costos que la cartera $A$ impone al agente en términos de mayor esfuerzo ejercido y mayor riesgo soportado.

\subsection{Robustez de los resultados}

Tres aspectos relacionados con la robustez de los resultados principales del artículo son los siguientes: (i) actitud del principal frente al riesgo, (ii) plausibilidad del incumplimiento de la condición de MLRP y (iii) número de carteras disponibles para el agente. ${ }^{16}$ Mientras los dos primeros aspectos son abordados a continuación, el tema del espacio de carteras de inversión es discutido en la siguiente sección.

Actitud del principal frente al riesgo. Note que nuestros resultados son válidos bajo el supuesto de neutralidad al riesgo por parte del dueño del fondo. En efecto, en un resultado clásico, la teoría de contratos sostiene que si el principal y el agente son aversos al riesgo, el contrato óptimo debe ser tal que ambos soporten parte del riesgo involucrado en la relación contractual (en este caso el riesgo de los retornos de las carteras) en proporción a sus grados de aversión al riesgo. En el contexto de la Proposición 2, esto significa que debemos considerar dos elementos adicionales para garantizar que el dueño del fondo efectivamente preferirá la cartera $A$ sobre la $B$. En primer lugar, desde el punto de vista del principal, ya no bastará con centrarnos en los retornos esperados de ambas carteras, sino además debemos tomar en cuenta la volatilidad de éstas. En segundo lugar, no sólo debemos tomar en consideración el grado de aversión al riesgo del trader, sino también aquél del dueño del fondo.

Sin embargo, no podemos afirmar a priori que estas consideraciones adicionales harán demasiado exigentes las nuevas condiciones que permitan garantizar que el principal prefiera la cartera $A$ sobre la $B$. Esto se debe a que si bien ahora el dueño del fondo también toma en cuenta la volatilidad de las carteras, él puede traspasar parte de ese riesgo al trader en una proporción que dependerá de los grados de aversión de ambas partes.

15 Esta propiedad en términos de valores esperados es garantizada por la dominancia estocástica de primer orden de la cartera $A$ sobre la $B$. No obstante, como el Corolario 1 establece, el incumplimiento de esta última propiedad garantiza a su vez un esquema de compensación no monótono.

16 Agradecemos a los árbitros por sugerir esta discusión. 
Plausibilidad del incumplimiento de la condición de MLRP. Es importante notar que muchas distribuciones de probabilidad tradicionales satisfacen el supuesto de MLRP, como la distribución normal y aquéllas pertenecientes a la familia de la distribución exponencial (Milgrom, 1981). Sin embargo, como discuten e ilustran Grossman y Hart (1983, p. 24) y Caillaud y Hermalin (2000, p. 23), es bastante sencillo encontrar ejemplos razonables de distribuciones que no cumplen esta propiedad, y en los que un esquema de incentivos no monótono emerge como una solución aceptable. La razón principal para la plausibilidad del incumplimiento de la condición de MLRP se basa en que, en un ambiente de asimetría de información, el resultado de la relación principal-agente cumple una dualidad de funciones. Por una parte, el resultado mejora directamente el bienestar del principal porque le permite acceder a mayores niveles de consumo. Adicionalmente, el resultado sirve también al principal como un instrumento para inferir el esfuerzo (no observado) del agente. En ciertas circunstancias, esos dos roles pueden entrar en conflicto, haciendo la violación de la condición MLRP totalmente razonable desde un punto de vista económico (ver Grossman y Hart, 1983, p. 9 y p. 28).

En relación con este último punto, podemos agregar un resultado de carácter técnico. El siguiente corolario presenta una condición más fuerte (pero tal vez más intuitiva) que el incumplimiento de la MLRP, que también permite un esquema compensatorio no monótono.

Corolario 1. Si existe un par de retornos $x_{i}, x_{i+1}<x_{n}$, tal que

$$
\sum_{j=1}^{i} p_{j}^{A} \geq \sum_{j=1}^{i} p_{j}^{B},
$$

entonces $w\left(x_{i}\right) \geq w\left(x_{i+1}\right)$.

Por tanto, una condición suficiente adicional para que una estructura remunerativa óptima premie bajos retornos es que la cartera $A$ no exhiba una dominancia estocástica de primer orden sobre la cartera $B .{ }^{17}$

Concluimos esta sección retornando brevemente al ejemplo de la sección 2 , de tal modo de verificar si las condiciones para esquemas de incentivos no monótonos caracterizadas por el modelo teórico se cumplen. En primer lugar, observemos que en este ejemplo efectivamente no se satisface la propiedad de MLRP. En particular, para $x_{2}>x_{1}$, tenemos que:

$$
L R_{2}=\frac{3}{10}<L R_{1}=\frac{12}{5}
$$

y, por tanto, aplicando la Proposición 1, debe verificarse que $w\left(x_{2}\right)<w\left(x_{1}\right)$.

17 El ejemplo presentado en la sección 2 ilustra precisamente esta situación. Note, sin embargo, que el incumplimiento de la dominancia estocástica de primer orden no impide que el retorno esperado de la cartera $A$ supere a aquél de la cartera $B$, como dicho ejemplo muestra. Para una discusión más formal, ver Shaked y Shanthikumar (1994). 
En segundo lugar, en este ejemplo tampoco se verifica la propiedad de dominancia estocástica de primer orden en favor de ninguna de las dos carteras. En efecto, note que si bien $\sum_{i=1}^{2} p_{i}^{A}=3 / 5<\sum_{i=1}^{2} p_{i}^{B}=5 / 6$, también se cumple que $p_{1}^{A}=2 / 5>1 / 6=p_{1}^{B}$. Por consiguiente, podemos aplicar el Corolario 1 , el que también garantiza un perfil de compensaciones no monótono.

\section{Extensión: Nivel de Esfuerzo No Binario}

\subsection{Discusión}

Con respecto al supuesto acerca del espacio de los niveles de esfuerzo (equivalente al espacio de carteras disponibles en nuestro modelo), destacamos que el artículo intenta adoptar la estructura más simple posible para mostrar la posibilidad de no monotonicidades en el esquema compensatorio. En esa línea, hasta ahora hemos asumido que los niveles de esfuerzo son sólo dos (y por tanto también el número de carteras disponibles). Para conjeturar qué ocurriría si considerásemos un número mayor de opciones de carteras (pero aún finito), podemos remitirnos a la literatura previa. Esta ha mostrado que cuando el esfuerzo es una variable no binaria, la monotonicidad del plan de incentivos requiere de supuestos adicionales, y puede ser asegurada de dos maneras: (i) con el cumplimiento conjunto de la condición de MLRP y el requisito de una función de distribución cóncava (CDFP) (Mirrlees, 1979), o (ii) la propiedad de spanning condition (SC) (Grossman y Hart, 1983). Por consiguiente, en este caso el conjunto de condiciones que permitirían que dicha monotonicidad no se cumpla sería más grande. En particular, incluso si la condición de MLRP se verificase, igualmente puede llegar a ser óptimo un esquema de incentivos que incluya un "premio al fracaso" (un ejemplo de esta situación es presentado en Grossman y Hart, 1983, p. 24).

Por otra parte, si considerásemos opciones no finitas de carteras, estaríamos en un ambiente en que el nivel de esfuerzo sería una variable continua. Caillaud y Hermalin (2000, p. 47) plantean que en este marco de modelamiento un esquema de incentivos monótonos también requiere de la propiedad de spanning. Al ser esta última una condición más fuerte que MLRP, podemos concluir que en este caso la posibilidad de no monotonicidades también podría ser más alta que lo que el modelo con esfuerzo binario admite.

En general, la literatura de teoría de contratos muestra que, al sofisticar el modelo básico de riesgo moral en relación al espacio de acciones del agente, sólo se complejiza el análisis en términos técnicos, pero no se alteran sustancialmente los resultados en términos cualitativos. ${ }^{18}$ Dado que nuestro modelo es una adaptación muy simple del problema de riesgo moral clásico, podemos conjeturar entonces que aunque pueden emerger condiciones adicionales para esquemas de incentivos no monótonos al extender el espacio de niveles de esfuerzo, las condiciones halladas en las secciones anteriores permanecen siendo válidas. Por

18 Ver Caillaud y Hermalin (2000) para una discusión más detallada sobre la conveniencia de un modelo finito y más parsimonioso. 
tanto, el mensaje central del artículo no parece depender crucialmente de si el número de opciones de carteras es finito o no.

Para reforzar esta conjetura, a continuación presentamos un modelo sencillo en el que aunque el nivel de esfuerzo (grado de innovación de la cartera de inversión) es ahora una variable continua, el incumplimiento de la propiedad MLRP continúa siendo una condición suficiente para un esquema de incentivos no monótono.

\subsection{Un modelo con esfuerzo continuo}

Este modelo conserva la mayoría de los supuestos adoptados en la sección 3 con respecto al proceso de delegación de administración de carteras de inversión. La principal diferencia radica en que ahora la variable $e$ representa el grado de innovación de la cartera de inversión que el trader puede escoger. Asumimos que el espacio de esta variable de decisión es continuo tal que $e \in[0,1]$. El grado de innovación puede ser visto como la ponderación $e$ que el trader pone sobre tecnologías, procedimientos y estrategias innovadores. Por contrapartida, $(1-e)$ puede ser entendido como la ponderación puesta por el trader sobre tecnologías, procedimientos y estrategias más convencionales. ${ }^{19}$

En este contexto, definimos $x_{i}^{e}$ como el retorno generado por una cartera de inversión con un grado de innovación $e$ cuando ocurre el estado de la naturaleza $i$. Asimismo, denotamos por $p_{i}^{e}$ a la probabilidad de ocurrencia del retorno $x_{i}^{e}$, tal que se verifica que $p_{i}^{e}>0$ para todo $i, e, \mathrm{y} \sum_{i=1}^{n} p_{i}^{e}=1$. Por simplicidad, suponemos que existen tres estados de la naturaleza $(n=3)$, tal que $x_{i}^{e} \in X=\left\{x_{1}, x_{2}, x_{3}\right\}$ para todo $e$, y que $x_{1}<x_{2}<x_{3} \cdot{ }^{20}$ En términos más concretos, suponemos que

$$
p_{i}^{e}=\left\{\begin{array}{ccc}
\gamma_{1} e & \text { si } & x=x_{1} \\
\left(\gamma_{2}-1\right) e+1 & \text { si } & x=x_{2} \\
\gamma_{3} e & \text { si } & x=x_{3}
\end{array}\right.
$$

donde $\gamma_{i}>0$ para todo $i$ y $\sum_{i=1}^{3} \gamma_{i}=1$. Note que estas restricciones sobre los parámetros $\gamma^{\prime} s$ permiten que $\left(\partial p_{i}^{e} / \partial e\right)>0$ para $i=1 \mathrm{y} i=3$, pero $\left(\partial p_{i}^{e} / \partial e\right)<0$ para $i=2$. Por tanto, el hecho de que un $e$ más alto incre-

19 De acuerdo a la terminología financiera, podemos interpretar $e$ como el grado en que una estrategia de inversión es "activa", es decir, se aleja de una cartera de referencia, como por ejemplo, un índice accionario.

20 Con el objeto de aislar los efectos de considerar un espacio continuo de acciones, simplificamos el análisis respecto del número de estados de la naturaleza posibles. Por otra parte, un espacio continuo de estados de la naturaleza no afecta las propiedades de monotonicidad del esquema óptimo de incentivos (para una discusión más formal, ver Caillaud y Hermalin, 2000). 
mente la probabilidad de los eventos extremos es consistente con la idea de que $e$ parametriza el riesgo de la cartera de inversión.

Así también, esta formulación implica que la propiedad de MLRP no se cumple. En efecto, si definimos el likelihood ratio como

$$
L R_{i}=\frac{\partial p_{i}^{e} / \partial e}{p_{i}^{e}}
$$

es sencillo verificar que $L R_{1}>L R_{2}{ }^{21}$

El principal es neutral al riesgo y las preferencias del agente están descritas por la siguiente función de utilidad aditivamente separable:

$$
U(w, e)=u(w)-v(e) .
$$

La función $u(\cdot)$ conserva las mismas propiedades descritas en la sección 2. Por otra parte, dado que asumimos que un mayor grado de innovación requiere un mayor nivel de esfuerzo, suponemos que la función de desutilidad $v(\cdot)$ satisface $v_{e}^{\prime}>0$ (desutilidad marginal positiva) $\mathrm{y} v_{e}^{\prime \prime}>0$ (desutilidad marginal creciente). ${ }^{22}$

Además, asumimos que en un ambiente con asimetría de información, la decisión óptima del principal nunca es evitar carteras riesgosas (innovadoras). Formalmente, asumimos que:

$$
\sum_{i=1}^{3} p^{e^{*}}{ }_{i} x^{e^{*}}{ }_{i}-x_{2}>\sum_{i=1}^{3} p^{e^{*}}{ }_{i} w^{*}\left(x_{i}\right)-w^{*}(0)
$$

donde $w^{*}(0)$ representa el salario asociado al contrato de primer mejor que induce un grado de innovación $e=0 \mathrm{y} w^{*}\left(x_{i}\right)$ representa el salario asociado al contrato de segundo mejor que induce un grado de innovación óptimo $e^{*} \in(0,1]$. Note que este supuesto es análogo a la condición enunciada en la Proposición 2 para el caso del modelo con esfuerzo binario.

Luego, si el principal desea implementar el grado de innovación $e_{0} \in(0,1]$, el contrato óptimo resuelve el siguiente problema:

$$
\underset{\left\{w\left(x_{i}\right)\right\}_{i=1}^{3}}{\operatorname{Min}} \sum_{i=1}^{3} p_{i}^{e_{0}} w\left(x_{i}\right)
$$

s.a.

$21 \quad$ En efecto, $L R_{2}=\frac{1}{e+\left(1 / \gamma_{2}-1\right)}<0<\frac{1}{e}=L R_{1}$.

22 Este supuesto parece consistente con lo observado en la práctica en los mercados financieros. En efecto, las administradoras de fondos mutuos suelen cobrar por estrategias de inversión activas comisiones más altas que por estrategias de inversión pasivas. 


$$
\begin{gathered}
\sum_{i=1}^{3} p_{i}^{e_{0}} u\left(w\left(x_{i}\right)\right)-v\left(e_{0}\right) \geq \underline{U} \\
e_{0}=\arg \max _{e} \sum_{i=1}^{3} p_{i}^{e} u\left(w\left(x_{i}\right)\right)-v(e),
\end{gathered}
$$

donde (5.3) representa la restricción de participación y (5.4), la restricción de compatibilidad de incentivos. Aplicando el enfoque denominado First Order Approach (Rogerson, 1985), podemos remplazar la segunda restricción por la condición de primer orden del problema de esfuerzo óptimo del agente. ${ }^{23}$ Esta condición está dada por:

$$
\gamma_{3} u\left(w\left(x_{3}\right)\right)-\left(1-\gamma_{2}\right) u\left(w\left(x_{2}\right)\right)+\gamma_{1} u\left(w\left(x_{1}\right)\right)-v^{\prime}\left(e_{0}\right)=0
$$

Asimismo, es sencillo verificar que la restricción de participación se cumple con igualdad en el óptimo:

$$
\sum_{i=1}^{3} p_{i}^{e_{0}} u\left(w\left(x_{i}\right)\right)-v\left(e_{0}\right)=\underline{U} .
$$

Combinando (5.5) y (5.6) obtenemos que:

$$
w^{0}\left(x_{2}\right)=u^{-1}\left(\underline{U}+v\left(e_{0}\right)-e_{0} v^{\prime}\left(e_{0}\right)\right) \equiv u^{-1}\left(u_{2}\right),
$$

a partir de lo cual es posible verificar que:

$$
\frac{\partial w^{0}\left(x_{2}\right)}{\partial e_{0}}=-\frac{1}{u^{\prime}\left(u^{-1}\left(u_{2}\right)\right)} e_{0} v^{\prime \prime}\left(e_{0}\right)<0
$$

Por tanto, en la medida que el dueño del fondo mutuo desee invertir en una cartera con un mayor grado de innovación, el contrato contendrá una menor compensación para el trader en caso de observar un retorno intermedio $x=x_{2}$.

Al sustituir (5.7) en la restricción (5.5), tenemos que:

$$
\gamma_{3} u\left(w\left(x_{3}\right)\right)-\left(1-\gamma_{2}\right) u\left(u^{-1}\left(u_{2}\right)\right)+\gamma_{1} u\left(w\left(x_{1}\right)\right)-v^{\prime}\left(e_{0}\right)=0 .
$$

$\overline{23}$ Note que la concavidad de $p_{i}^{e}$ garantiza la validez del enfoque de primer orden. 
De esta última ecuación obtenemos:

$$
w\left(x_{3}\right)=u^{-1}\left[\frac{\left(1-\gamma_{2}\right) u\left(u^{-1}\left(u_{2}\right)\right)-\gamma_{1} u\left(w\left(x_{1}\right)\right)+v^{\prime}\left(e_{0}\right)}{\gamma_{3}}\right] \equiv u^{-1}\left(u_{3}\right) .
$$

Luego, el programa que resuelve el contrato óptimo se reduce a:

$$
\underset{w\left(x_{1}\right)}{\operatorname{Min}} \gamma_{1} w\left(x_{1}\right)+\gamma_{3} u^{-1}\left(u_{3}\right)
$$

La condición de primer orden de este problema es:

$$
\gamma_{1}+\gamma_{3} \frac{1}{u^{\prime}\left(u^{-1}\left(u_{3}\right)\right)} \frac{\partial u_{3}}{\partial w\left(x_{1}\right)}=\gamma_{1}-\gamma_{3} \frac{1}{u^{\prime}\left(u^{-1}\left(u_{3}\right)\right)} \frac{\gamma_{1}}{\gamma_{3}} u^{\prime}\left(w\left(x_{1}\right)\right)=0 .
$$

Esta última igualdad implica que:

$$
u^{\prime}\left(w\left(x_{1}\right)\right)=u^{\prime}\left(u^{-1}\left(u_{3}\right)\right)=u^{\prime}\left(w\left(x_{3}\right)\right) .
$$

Dado que la función $u^{\prime}(\cdot)$ es estrictamente decreciente, la última ecuación implica que:

$$
w^{0}\left(x_{1}\right)=w^{0}\left(x_{3}\right)
$$

lo que significa que el esquema remunerativo óptimo exhibe una clara nomonotonicidad al asignar la misma compensación al trader cuando el retorno observado es bajo $\left(x_{1}\right)$ o alto $\left(x_{3}\right)$. Tras sustituir (5.10) en (5.8), obtenemos que:

$$
\gamma_{3} u\left(w\left(x_{1}\right)\right)=v^{\prime}\left(e_{0}\right)-\gamma_{1} u\left(w\left(x_{1}\right)\right)+\left(1-\gamma_{2}\right) u\left(u^{-1}\left(u_{2}\right)\right)
$$

Resolviendo para $u\left(w\left(x_{1}\right)\right)$, encontramos que:

$$
\begin{aligned}
w^{0}\left(x_{3}\right)=w^{0}\left(x_{1}\right) & =u^{-1}\left[\frac{v^{\prime}\left(e_{0}\right)}{1-\gamma_{2}}+u\left(u^{-1}\left(u_{2}\right)\right)\right] \\
& =u^{-1}\left[\frac{v^{\prime}\left(e_{0}\right)}{1-\gamma_{2}}+u\left(w^{0}\left(x_{2}\right)\right)\right] .
\end{aligned}
$$

De esta última expresión es sencillo comprobar que dado que $v^{\prime}\left(e_{0}\right) /\left(1-\gamma_{2}\right)>0 \mathrm{y} u^{-1}(\cdot)$ es una función estrictamente creciente, se verifica que: 


$$
w^{0}\left(x_{3}\right)=w^{0}\left(x_{1}\right)>w^{0}\left(x_{2}\right)
$$

Esto implica que el esquema óptimo de incentivos incluye un premio $e x$ ante al fracaso que busca alentar la conformación de carteras de inversión más innovadoras (riesgosas). Adicionalmente, note que diferenciando (5.11) con respecto a $e_{0}$ tenemos que:

$$
\operatorname{sign} \frac{\partial w^{0}\left(x_{3}\right)}{\partial e_{0}}=\operatorname{sign} \frac{\partial w^{0}\left(x_{1}\right)}{\partial e_{0}}=\operatorname{sign}\left(\frac{1}{1-\gamma_{2}}-e_{0}\right)>0
$$

Por tanto, en la medida que el dueño del fondo mutuo desee invertir en una cartera con un mayor grado de innovación, el contrato contendrá una mayor compensación para el trader en caso de observar los retornos extremos $x_{1}$ y $x_{3}$.

En suma, el modelo desarrollado en esta sección ilustra cómo los resultados obtenidos por un modelo con esfuerzo binario (como aquél desarrollado en las secciones 3 y 4) se mantienen cuando el esfuerzo es una variable continua. En particular, se mantiene la validez de las conclusiones de la Proposición 1 en el sentido de que la violación de la MLRP continúa siendo una condición suficiente para observar no monotonicidades en el esquema óptimo de incentivos. ${ }^{24}$

\section{Conclusiones}

Este artículo estudia, en el contexto de la administración delegada de carteras, el diseño de esquemas de incentivos óptimos para ejecutivos. En particular, se caracterizan las condiciones bajo las cuales dichos esquemas admiten una relación no monótona entre la compensación pagada a los ejecutivos y el desempeño de las carteras bajo su administración.

Estas condiciones están relacionadas con los incentivos que el fondo de inversión debe proveer a sus ejecutivos para estimularlos a que exploren y propongan carteras de inversión innovadoras, y para desalentarlos a que seleccionen carteras conservadoras o simplemente repliquen la cartera de mercado. Con este objeto, es posible que el contrato óptimo deba premiar rentabilidades extremas, ya sean éstas suficientemente altas o suficientemente bajas. En consecuencia, una suerte de "premio al fracaso" podría emerger como un elemento válido en el esquema óptimo de incentivos.

Por cierto, una política indiscriminada de premios al fracaso puede generar incentivos perversos que desemboquen en asunciones excesivas de riesgo, como aquellas observadas en el origen de la actual crisis financiera. Una primera extensión natural de este trabajo es, por tanto, la inclusión de estrategias de inversión demasiado riesgosas que permitan matizar los resultados aquí obte-

24 El resultado enunciado en la Proposición 2 también se mantiene. En efecto, el supuesto descrito por la ecuación (5.1) no es más que la extensión de la condición caracterizada en dicha proposición para el caso en que el esfuerzo es una variable continua. 
nidos, y conlleven un esquema de compensación que desincentive conductas fraudulentas y de risk-shifting.

Por último, reconocemos las limitantes de nuestras conclusiones debido al carácter básico del modelo de riesgo moral adoptado en este artículo. En consecuencia, este modelo debe ser interpretado como un marco de referencia sobre el cual se pueden adicionar elementos más realistas en el modelamiento de los esquemas de incentivos de ejecutivos. En este sentido, una segunda extensión del trabajo apunta a someter la robustez de nuestros resultados a elementos que influyen en la relación contractual, y que han sido incorporados profusamente por la literatura de compensación de ejecutivos durante los últimos 20 años, tales como la selección adversa, la sustitución entre incentivos explícitos e implícitos, y la no completitud de los contratos.

\section{REFERENCIAS}

Admati, A.R. y Pfleiderer, P. (1997). “Does It All Add Up? Benchmarks and the Compensation of Active Portfolio Managers", Journal of Business, 70 (3), 323-350.

Bolton, P. y Dewatripont, M. (2005). Contract Theory, The MIT Press.

Cadenillas, A., Cvitanic, J. y Zapatero, F. (2007). “Optimal Risk-Sharing with Effort and Project Choice", Journal of Economic Theory, 133 (1), 403440.

Caillaud, B. y Hermalin, B.E. (2000). "Hidden Action and Incentives", Technical Notes, Ecole Ponts et Chaussée, Paris, y University of California, Berkeley.

Carpenter, J.N. (2000). “Does Option Compensation Increase Managerial Risk Appetite?", Journal of Finance, 55 (5), 2311-2331.

Chen, H.L. y Pennacchi, G. (2009). "Does Prior Performance Affect a Mutual Fund's Choice of Risk? Theory and Further Empirical Evidence", Journal of Financial and Quantitative Analysis, 44 (4), 745-775.

Dybvig, P.H.; Farnsworth, H.K. y Carpenter, J.N. (2010). "Portfolio Performance and Agency", The Review of Financial Studies, 23 (1), 1-23.

Garcia, D. (2001). "Optimal Menus of Linear Contracts in Portfolio Selection Models", Working Paper, Tuck School of Business Administration.

Goetzmann, W.N.; Ingersoll, J. y Ross, S.A. (2003). "High Water Marks and Hedge Funds Management Contracts", Journal of Finance, 58 (4), 1685-1718.

Grossman, S.J. y Hart, O.D. (1983). "An Analysis of the Principal-Agent Problem", Econometrica, 51 (1), 7-45.

Gruber, M. (1996). “Another Puzzle: The Growth of Actively-Managed Mutual Funds", Journal of Finance, 51, 783-810.

Heinkel, R. y Stoughton, N.M. (1994). "The Dynamics of Portfolio Management Contracts", The Review of Financial Studies, 7, 351-87.

Holmström, B. (1979). "Moral Hazard and Observability", Bell Journal of Economics, 10 (1), 74-91.

Hvide, H.K. (2002). "Tournament Rewards and Risk Taking”, Journal of Labor Economics, 20 (4), 877-898. 
Innes, R.D. (1990). "Limited Liability and Incentive Contracting with Ex-Ante Action Choices", Journal of Economic Theory, 52, 45-67.

Li, T. y Zhou, Y. (2006). "Optimal Contracts in Portfolio Delegation", Working Paper, The Chinese University of Hong Kong.

Malkiel, B.G. (1995). "Returns from Investing in Equity Mutual Funds 1971 to 1991", Journal of Finance, 50 (2), 549-572.

Manso, G. (2010). "Motivating Innovation”, Working Paper, MIT Sloan School of Management.

Milgrom, P.R. (1981). "Good News and Bad News: Representation Theorems and Applications", Bell Journal of Economics, 12, 380-391.

Mirrlees, J.A. (1976). “The Optimal Structure of Incentives and Authority Within an Oganization”, Bell Journal of Economics, 7, 105-131.

Mirrlees, J.A. (1979). "The Implications of Moral Hazard for Optimal Insurance”, Seminario ofrecido en la Conferencia en Honor a Karl Borch, Bergen, Noruega, Mimeo.

Ou-Yang, O. (2003). "Optimal Contracts in a Continuous-Time Delegated Portfolio Management Problem", The Review of Financial Studies, 16 (1), 173-208.

Rogerson, W.P. (1985). "The First-Order Approach to Principal-Agent Problems", Econometrica, 53, 1357-1367.

Ross, S.A. (2004). "Compensation, Incentives and the Duality of Risk Aversion and Riskiness", Journal of Finance, 59 (1), 207-225.

Shaked, M. y Shanthikumar, J.G. (1994). Stochastic Orders and Their Applications, San Diego: Academic Press.

Starks, L.T. (1987). "Performance Incentive Fees: An Agency Theoretic Approach", Journal of Financial and Quantitative Analysis, 22 (1), 17-32.

Stoughton, N.M. (1993). "Moral Hazard and the Portfolio Management Problem", Journal of Finance, 48 (5), 2009-2028.

Stracca, L. (2006). "Delegated Portfolio Management: A Survey of the Theoretical Literature", Journal of Economic Surveys, 20 (5), 823-848. 


\section{ANEXOS}

\section{Anexo A: Pruebas}

Prueba de la Proposición 1. El Lagrangiano de este problema corresponde a:

$$
\begin{aligned}
L\left(\left\{w\left(x_{i}\right)\right\}, \lambda, \mu\right)= & \sum_{i=1}^{n} p_{i}^{A}\left[x_{i}-w\left(x_{i}\right)\right] \\
& +\lambda\left[\sum_{i=1}^{n} p_{i}^{A} u\left(w\left(x_{i}\right)\right)-v\left(e_{h}\right)-\underline{\mathrm{U}}\right] \\
& +\mu\left[\sum_{i=1}^{n}\left[p_{i}^{A}-p_{i}^{B}\right] u\left(w\left(x_{i}\right)\right)-v\left(e_{h}\right)+v\left(e_{l}\right)\right]
\end{aligned}
$$

donde $\lambda$ y $\mu$ corresponden a los multiplicadores de Lagrange asociados a las restricciones (4.2) y (4.3), respectivamente. De las condiciones de primer orden de este problema, obtenemos:

$$
\frac{1}{u^{\prime}\left(w\left(x_{i}\right)\right)}=\lambda+\mu\left(1-\frac{1}{L R_{i}}\right)
$$

Aplicando las condiciones de Karush-Kuhn-Tucker y argumentos tradicionales de la teoría de contratos (ver por ejemplo Bolton y Dewatripont, 2005), es posible verificar que ambas restricciones están activas, y por tanto $\lambda, \mu>0$. En particular, si la restricción de incentivos está activa, de (1) es fácil advertir que los pagos varían con el resultado: $w\left(x_{i}\right)$ varía directamente con $L R_{i}$.

De (1) obtenemos:

$$
w\left(x_{i}\right)=u^{\prime-1}\left(\frac{1}{\lambda+\mu\left(1-\frac{1}{L R_{i}}\right)}\right)
$$

Dado que el agente es averso al riego, $u^{\prime}(\cdot)$ es una función decreciente, y por ende, $u^{-1}(\cdot)$ también lo es. Además, dado que $\mu>0$, todo lo anterior implica que:

$$
\operatorname{sign}\left(w\left(x_{i+1}\right)-w\left(x_{i}\right)\right)=\operatorname{sign}\left(L R_{i+1}-L R_{i}\right),
$$

lo que completa la prueba. Q.E.D. 
Prueba de la Proposición 2. Primero, si el principal desea que el agente seleccione la cartera $B$, y por tanto implementar el esfuerzo bajo $e_{i}$, la compensación óptima corresponde a la compensación óptima bajo simetría de información para ese nivel de esfuerzo. Es decir, $w^{*}\left(x_{i}\right)=w^{*}\left(e_{l}\right)=u^{-1}\left(\underline{U}+v\left(e_{l}\right)\right)$ para todo $i$ a partir de la restricción de participación activa. Segundo, de lo anterior y (4.1), es posible inferir que el principal preferirá la cartera $A$ sobre la $B$, si

$$
E\left(x-w^{*}(x) \mid e_{h}\right)>E\left(x \mid e_{l}\right)-w^{*}\left(e_{l}\right),
$$

lo cual, dada la relación entre los niveles de esfuerzo y las carteras y debido a que $E(\cdot)$ es un operador lineal, es equivalente a:

$$
\sum_{i=1}^{n}\left(p_{i}^{A}-p_{i}^{B}\right) x_{i}>\sum_{i=1}^{n} p_{i}^{A} w^{*}\left(x_{i}\right)-w^{*}\left(e_{l}\right),
$$

lo que completa la prueba. Q.E.D.

Prueba del Corolario 1. Siguiendo a Shaked y Shanthikumar (1994), note que una condición suficiente para el incumplimiento de la propiedad de MLRP es que la distribución de probabilidad de los retornos de la cartera $A$ no exhiba una dominancia estocástica de primer orden sobre aquella de la cartera $B$. En el contexto del modelo, esta condición corresponde a aquella enunciada en el corolario. Luego, aplicando la Proposición 1, el resultado del corolario queda establecido. Q.E.D.

\section{ANEXo B: Contrato óPTIMO BAJO SIMETría de INFORMACión}

En este anexo caracterizamos el contrato óptimo del ejemplo descrito en la sección 2, bajo un ambiente de simetría de información. En este contexto, si el principal es neutral al riesgo y el agente es averso al riesgo, el primero asegura completamente al segundo. Por tanto, se verifica que $w\left(x_{i}\right)=w$ para todo $i$. De la restricción de participación activa se cumple que $w=w(e)=e^{4}$. Es posible verificar que con el nivel de esfuerzo consistente con el portafolio $A$ (es decir, $e=3$ ), el principal obtiene una utilidad esperada de

$$
\begin{aligned}
E(x-w(e) \mid e=3) & =E(x \mid e=3)-w(e=3) \\
& =200-81 \\
& =119 .
\end{aligned}
$$

Por otra parte, con el nivel de esfuerzo necesario para el portafolio $B$ (es decir, $e=1$ ), el principal obtiene una utilidad esperada de 


$$
\begin{aligned}
E(x-w(e) \mid e=1)=E(x \mid e & =1)-w(e=1) \\
& =83,3-1 \\
& =82,3
\end{aligned}
$$

Esto implica que el principal prefiere que el agente seleccione la cartera $A$, y, por lo tanto, el contrato óptimo es:

$$
w^{*}=\left\{\begin{array}{lll}
81 & \text { si } & e=3 \\
(-\infty, 1) & \text { si } & e=1
\end{array}\right. \text {. }
$$

